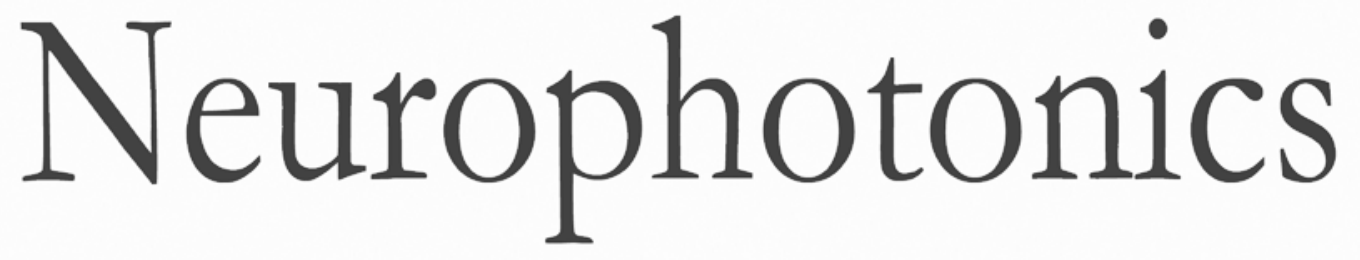

\title{
Review of transcranial photobiomodulation for major depressive disorder: targeting brain metabolism, inflammation, oxidative stress, and neurogenesis
}

Paolo Cassano

Samuel R. Petrie

Michael R. Hamblin

Theodore A. Henderson

Dan V. Iosifescu 


\title{
Review of transcranial photobiomodulation for major depressive disorder: targeting brain metabolism, inflammation, oxidative stress, and neurogenesis
}

\author{
Paolo Cassano, ${ }^{a, b, \star}$ Samuel R. Petrie, ${ }^{a}$ Michael R. Hamblin, ${ }^{c, d, e}$ Theodore A. Henderson, ${ }^{\text {f,g }}$ and Dan V. losifescu ${ }^{\text {,i }}$ \\ ${ }^{a}$ Massachusetts General Hospital, Depression Clinical and Research Program, One Bowdoin Square, 6th Floor, Boston, Massachusetts 02114, \\ United States \\ bHarvard Medical School, Department of Psychiatry, 401 Park Drive, Boston, Massachusetts 02215, United States \\ 'Massachusetts General Hospital, Wellman Center for Photomedicine, 50 Blossom Street, Boston, Massachusetts 02114, United States \\ dHarvard Medical School, Department of Dermatology, 55 Fruit Street, Boston, Massachusetts 02114, United States \\ EHarvard-MIT Division of Health Sciences and Technology, 77 Massachusetts Avenue, Cambridge, Massachusetts 02139, United States \\ 'Synaptic Space, 3979 East Arapahoe Road, Littleton, Colorado 80122, United States \\ 9Neuro-Laser Foundation, Suite 420, 215 South Wadsworth, Lakewood, Colorado 80226, United States \\ hMount Sinai Medical School, Mood and Anxiety Disorders Program, 1428 Madison Avenue, New York, New York 10029, United States \\ 'Mount Sinai Medical School, Department of Psychiatry and Neuroscience, 1 Gustave L. Levy Place, New York, New York 10029, United States
}

\begin{abstract}
We examined the use of near-infrared and red radiation (photobiomodulation, PBM) for treating major depressive disorder (MDD). While still experimental, preliminary data on the use of PBM for brain disorders are promising. PBM is low-cost with potential for wide dissemination; further research on PBM is sorely needed. We found clinical and preclinical studies via PubMed search (2015), using the following keywords: "near-infrared radiation," "NIR," "low-level light therapy," "low-level laser therapy," or "LLLT" plus "depression." We chose clinically focused studies and excluded studies involving near-infrared spectroscopy. In addition, we used PubMed to find articles that examine the link between PBM and relevant biological processes including metabolism, inflammation, oxidative stress, and neurogenesis. Studies suggest the processes aforementioned are potentially effective targets for PBM to treat depression. There is also clinical preliminary evidence suggesting the efficacy of PBM in treating MDD, and comorbid anxiety disorders, suicidal ideation, and traumatic brain injury. Based on the data collected to date, PBM appears to be a promising treatment for depression that is safe and welltolerated. However, large randomized controlled trials are still needed to establish the safety and effectiveness of this new treatment for MDD. ๑ The Authors. Published by SPIE under a Creative Commons Attribution 3.0 Unported License. Distribution or reproduction of this work in whole or in part requires full attribution of the original publication, including its DOI. [DOI: 10.1117/1.NPh.3.3.031404]
\end{abstract}

Keywords: near-infrared radiation; photobiomodulation; low-level laser therapy; major depressive disorder; metabolism; inflammation; neurogenesis; oxidative stress; near-infrared; NILT; low-level light therapy.

Paper 15060SSVR received Dec. 28, 2015; accepted for publication Feb. 2, 2016; published online Mar. 4, 2016.

\section{Introduction}

Infrared (IR) light is ubiquitously present to most life on the earth. Of the total amount of solar energy reaching the human skin, $54 \%$ is IR and $30 \%$ is IR type A-near-infrared-(NIR; with a wavelength range of 760 to $1440 \mathrm{~nm}),{ }^{1}$ which penetrates through the human skin and reaches deeply into tissue, depending on wavelength and energy. ${ }^{2}$

NIR is used to treat a variety of conditions such as muscle pain, ${ }^{3}$ wounds, ${ }^{4}$ neuropathic pain, ${ }^{5}$ and headache. ${ }^{6}$ NIR is also used for wellness and lifestyle purposes such as for cosmetic improvement in peri-orbital wrinkles. ${ }^{7,8}$ The clinical use of NIR light applied in NIR-spectroscopy dates from the mid-1980s, when it was used for monitoring of the brain in the neonate and the fetus. ${ }^{9}$

The use of transcranial phototherapy for treating brain disorders started with its application to acute stroke. Numerous preclinical animal studies ${ }^{10-12}$ suggested that the application of NIR laser $(810 \mathrm{~nm})$ to the head at various times (hours) after induction of an acute stroke had beneficial effects on subsequent neurological performance and reduced lesion size. Evidence

*Address all correspondence to: Paolo Cassano, E-mail: pcassano@mgh harvard.edu was obtained for the anti-inflammatory, anti-apoptotic, and proneurogenesis effects in the brain stimulated by this approach. ${ }^{13,14}$ These promising animal studies led to the conduction of a series of clinical trials called NeuroThera Effectiveness and Safety Trials (NEST). All together there were three large studies conducted in 1410 stroke patients [NEST-1 $(n=120)$, NEST-2 $(n=660)$, NEST-3 $(n=630)]$ that demonstrated that NIR light delivered transcranially with a class-IV laser is safe, with no significant differences in rates of adverse events with NIR, when compared to sham exposure. ${ }^{15-17}$ Other preclinical studies and clinical trials have suggested that transcranial photobiomodulation (PBM: laser or light emitting diodes-LED) is safe and effective for acute ${ }^{18-22}$ and chronic ${ }^{23-25}$ traumatic brain injury (TBI) and has beneficial effects on neurodegenerative diseases (Alzheimer's and Parkinson's). ${ }^{26,27}$

For the transcranial treatment of major depressive disorder (MDD), both PBM LEDs and lasers have been experimentally tested, although PBM is not FDA-approved for the treatment of MDD. Certain forms of PBM treatment are also referred to as low-level light therapy (LLLT), since it utilizes light at a low power ( 0.1 to $0.5 \mathrm{~W}$ output at the source) to avoid any heating of tissue. The irradiance of the PBM medical devices (or power density) typically ranges from 1 to 10 times the NIR irradiance from sunlight on the skin $\left(33.6 \mathrm{~mW} / \mathrm{cm}^{2}\right.$ at the zenith). 
However, most PBM medical devices only deliver light energy at one or two selected wavelengths, as opposed to the whole spectrum of IR that is contained in sunlight. To our knowledge and to this date, transcranial PBM treatment has not caused any retinal injury - one of the most likely postulated adverse events, although care is taken routinely in such studies to protect the eyes with goggles or eye covers. ${ }^{28}$

In this review, we will first discuss the mechanisms of action by which NIR and red light (PBM) might improve symptoms of depression, and then present the clinical evidence for their use as a treatment for MDD and other comorbid psychiatric syndromes.

\section{Methods}

We found clinical and preclinical studies via PubMed search (December 15, 2015), using the following keywords: "nearinfrared radiation," "NIR," "low-level light therapy," "lowlevel laser therapy," or "LLLT" plus "depression." We chose studies that had a clinical focus, and we excluded studies involving NIR spectroscopy. We also located studies using the references from the articles found in the PubMed search. As the searched literature encompassed different conditions and disorders frequently comorbid with depression, a specific section of this review was devoted to the effect of PBM on psychiatric comorbidity. In the latter section, the following conditions were included, based on available literature: TBI, anxiety and post-traumatic stress syndromes, insomnia, and suicidal ideation. The literature search for the use of PBM to treat comorbid conditions was neither systematic nor extensive, but rather a secondary focus of this review. The information is presented in an organized fashion to allow the reader to easily grasp the potential applications of PBM for the treatment of depression and of its comorbid conditions. To attain this goal, the authors have allowed a margin of redundancy, by distributing different information derived from any given publication in separate sections of this review. To avoid an artificial inflation of the extant literature on the chosen topic, we referenced the main authors - and when appropriate their affiliation-when referring to the same articles more than once. The reader will find a table summarizing the six key clinical articles reviewed, also to avoid unintended inflation of the literature. The six clinical reports included in this review where extracted from a pool of 58 articles, that were originally identified with the literature search.

In addition, we used PubMed to find articles that examined the link between PBM and each of the various biological processes including metabolism, inflammation, oxidative stress, and neurogenesis.

\section{Targeting Brain Metabolism}

Multiple studies have reported regional and global hypometabolism in MDD, which could be related (either causally or consequentially) to the neurobiology of mood disorders. ${ }^{29-32}$ Positron emission tomography studies have shown abnormalities in glucose consumption rates and in blood flow in several brain regions of subjects with major depression. ${ }^{33}$ Moreover, metabolic abnormalities in the anterior cingulate, the amygdala-hippocampus complex, the dorsolateral prefrontal cortex (DLPFC), and inferior parietal cortex seem to improve after antidepressant treatment or after recovery. ${ }^{34-36}$

With phosphorus magnetic resonance spectroscopy ( $\left.{ }^{31} \mathrm{P}-\mathrm{MRS}\right)$, the baseline pool of nucleotide triphosphate (NTP) - a product of the cellular utilization of glucose and a marker of the cellular energy availability-was low in subjects who subsequently responded to antidepressant treatment. ${ }^{32}$ Iosifescu et al. ${ }^{32}$ also demonstrated for the first time with ${ }^{31} \mathrm{P}$-MRS a correlation between treatment response (to a regimen that combined antidepressants and triiodothyronine) and restoration of a higher NTP pool (with compensatory decrease in phosphocreatine) in the anterior cingulate cortex. This study suggests a pathway to antidepressant response based on restoration of a high cellular energy state. In fact, phosphocreatine represents a long-term storage depot of energy, while NTP and ATP are energy-rich molecules that are readily available to the cell. The same authors replicated the aforementioned findings in MDD subjects treated with standard antidepressants (Iosifescu et al., unpublished). In this cohort, ${ }^{31} \mathrm{P}$-MRS metabolite changes were noted in brain-only voxels of responders, but not in nonresponders to antidepressants.

In experimental and animal models, PBM (NIR and red light) noninvasively delivers energy to the cytochrome c oxidase and by stimulating the mitochondrial respiratory chain leads to increased ATP production (see Fig. 1). ${ }^{37-39}$ A study of the effects of NIR on patients with MDD found that a single session of NIR led to a marginally significant increase in regional cerebral blood flow. ${ }^{40}$ Whether the observed changes in cerebral blood flow resulted from fundamental changes in neuronal metabolism or changes in vascular tone remain to be clarified. Given the correlation of both hypometabolism and abnormal cerebral blood flow with MDD, the beneficial effect of NIR on brain metabolism is one potential mechanism for its antidepressant effect.

\section{Targeting Inflammation}

Animal and clinical research suggests that the inflammatory arm of the immune system contributes to MDD. Post-mortem gene expression profiling on tissue samples from Brodmann area 10

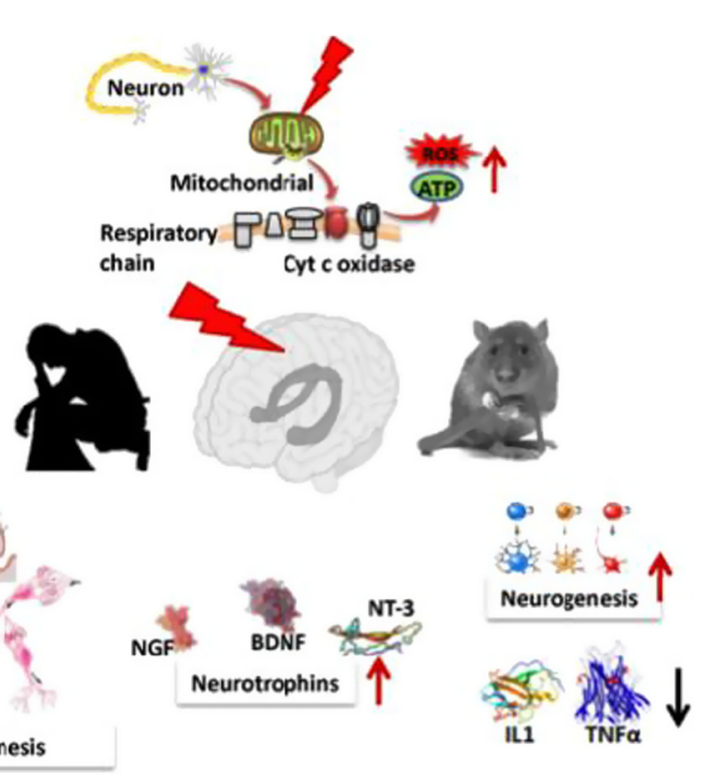

Fig. 1 Cellular targets of NIR radiation mechanisms of transcranial NIR for psychiatric disease. The NIR photons are absorbed by cytochrome $\mathrm{c}$ oxidase in the mitochondrial respiratory chain. This mitochondrial stimulation increases production of ATP but also activates signaling pathways by a brief burst of ROS. This signaling activates antioxidant defenses reducing overall oxidative stress. Proinflammatory cytokines and neuroinflammation are reduced. Neurotrophins such as brain-derived neurotrophic factor are upregulated, which in turn activate synaptogenesis (formation of new connections between existing neurons) and neurogenesis (formation of new neurons from neural stem cells). 
(BA10 - prefrontal cortex) have shown that MDD is characterized by increased inflammation and apoptosis. ${ }^{41}$ In a case-control study, Simon et al. ${ }^{42}$ found that antidepressant-naive MDD subjects had significant elevations in the following cytokines and chemokines when compared to healthy controls: MIP- $1 \alpha$, IL-1 $\alpha$, IL-1 $\beta$, IL-6, IL-8, IL-10, Eotaxin, GM-CSF, and IFN $\gamma$. Although IL-10 is an anti-inflammatory cytokine, the results suggested that the elevated levels of this IL-10 were likely induced in response to the overall elevation of proinflammatory cytokine levels. In a review of the research on inflammation in MDD, Raison et al. ${ }^{43}$ proposed that proinflammatory cytokines might cause brain abnormalities that are characteristic of MDD. Indeed, animal research has shown that IL-1 mediates chronic depression in mice by suppressing hippocampal neurogenesis. ${ }^{44}$

One proinflammatory cytokine that may be of particular relevance to depression is CSF IL-6 (IL6 measured in cerebrospinal fluid). In a recent report, patients with MDD had significantly higher CSF IL-6 levels compared to healthy controls; CSF IL-6 levels were significantly higher than in the serum, and there was no significant correlation between CSF and serum IL-6 levels. ${ }^{45}$ These findings are consistent with a prior report showing a positive correlation between CSF IL-6 levels and the severity of depression and suicide attempts, with the strongest correlation found in violent suicide attempters. ${ }^{46}$ One report in a smaller sample of depressed patients has shown that CSF IL- $6{ }^{47}$ was lower or comparable to healthy controls.

NIR light and red light (600 to $1600 \mathrm{~nm}$ ) decreased synovial IL-6 gene expression (decreased mRNA levels) in a rat model of rheumatoid arthritis. ${ }^{48}$ In another study, NIR $(810 \mathrm{~nm})$ used as a treatment for pain in patients with rheumatoid arthritis decreased production of the following proinflammatory cytokines: TNF- $\alpha$, IL- $1 \beta$, and IL- $8 .{ }^{49}$ Khuman et al. ${ }^{50}$ showed that transcranial NIR improved cognitive function and reduced neuroinflammation as measured by Iba1+ activated microglia in brain sections from mice that had suffered a TBI. Finally, NIR $(970 \mathrm{~nm})$ has been found to be an effective treatment for inflammatory-type acne. ${ }^{51}$ In summary, it is reasonable to predict that transcranial NIR treatment would likewise have an anti-inflammatory effect in patients suffering from MDD.

\section{Targeting Oxidative Stress}

Research has demonstrated a correlation between MDD and vulnerability to oxidative stress. ${ }^{52}$ For example, depression-induced rats show a significant decrease in glutathione peroxidase (GSH$\mathrm{Px}$ ) activity in the cortex. ${ }^{53}$ Glutathione (GSH) is the most abundant and one of the important antioxidants in the brain; GSH-Px enzymes protect against oxidative stress via reducing hydroperoxides and scavenging free radicals. ${ }^{54}$ GSH also appears reduced in the brains of MDD subjects. ${ }^{55}$ Additionally, a study by Sarandol et al. ${ }^{52}$ demonstrated that MDD patients have higher levels of malondialdehyde, a toxic molecule and a biomarker of oxidative stress. ${ }^{56}$ Moreover, depressed patients have more red blood cell (RBC) oxidation compared to healthy controls. ${ }^{52}$ In the same study, the authors found a significant positive correlation between RBC superoxide dismutase (SOD) activity and depression severity. SOD serves to catalyze the removal of the toxic superoxide radical. ${ }^{57}$ Thus, elevated SOD activity in depressed patients might indicate higher levels of oxidative stress. Finally, catalase activity and nitric oxide (NO) levels have also been shown to be lower in depressed patients than in healthy controls. ${ }^{58}$ Catalase is an enzyme that protects cells against damaging reactive oxygen species (ROS) via degradation of hydrogen peroxide to water and oxygen ${ }^{59} \mathrm{NO}$ has protective effects against cell damage, which are likely due to its pleiotropic functions in regulating antioxidant enzymes and many other aspects of cell metabolism. ${ }^{60,61}$

Oxidative stress may be an effective target for antidepressant treatments. However, successful treatments for MDD vary in regard to their protective effects against oxidative stress. ${ }^{52,53,62}$ Animal research suggests that PBM may have beneficial effects on oxidative stress. In a rat model of traumatized muscle, NIR (904 nm) blocked the release of harmful ROS and the activation of the transcription factor, nuclear factor $\kappa \mathrm{B}(\mathrm{NF}-\kappa \mathrm{B})$, both induced by muscle trauma. Trauma activates NF- $\kappa \mathrm{B}$ by destroying a specific protein inhibitor of NF- $\kappa \mathrm{B}$ called $\mathrm{I} \kappa \mathrm{B}$, and this destruction was inhibited by NIR light. Furthermore, NIR reduced the associated overexpression of the inducible form of nitric oxide synthase (iNOS) and reduced the production of collagen. ${ }^{63}$ This regulation of iNOS is important because excessive levels of iNOS can lead to formation of large amounts of NO that combine with superoxide radicals to form the damaging species peroxynitrite, and can interfere with the protective benefits of other forms of NO synthase. ${ }^{64}$ These findings suggest that NIR protects against oxidative stress induced by trauma. Finally, an in vitro study of the effects of red light and NIR (700 to $2000 \mathrm{~nm}$ ) on human RBCs found that NIR significantly protected RBCs against oxidation. ${ }^{65}$

\section{Targeting Neurogenesis}

A large body of research has demonstrated a link between stressinduced suppression of neurogenesis in the dentate gyrus (DG) and episodes of major depression. ${ }^{66-69}$ Additionally, MDD patients tend to exhibit significant hippocampal atrophy. ${ }^{70}$ Although there is a general consensus among reviewers that stress-induced suppression of hippocampal neurogenesis is not by itself a causal factor in the development of depression, ${ }^{71-73}$ studies demonstrate that many of the therapeutic effects of antidepressant treatments might be dependent on increasing DG neurogenesis. ${ }^{74,75}$

Animal research has shown that PBM stimulates neurogenesis and protects against cell death. Data suggest that red light, close to the NIR spectrum $(670 \mathrm{~nm})$, protects the viability of cell culture after oxidative stress, as indicated by mitochondria membrane potentials. ${ }^{74}$ NIR also stimulates neurite outgrowth mediated by nerve growth factor, and this effect could also have positive implications for axonal protection. ${ }^{76}$ Neuroprotective effects of red light $(670 \mathrm{~nm})$ were documented in in vivo models of mitochondrial optic neuropathy. ${ }^{77,78}$ Red light close to NIR spectrum $(670 \mathrm{~nm})$ has also been shown to protect neuronal cells against cyanide. ${ }^{79}$ In animal models of TBI, NIR $(810 \mathrm{~nm})$ appears to be an effective treatment and improves neurogenesis and synaptogenesis, via increase of brain-derived neurotrophic factor (BDNF). ${ }^{18,20,80-82}$ In addition, NIR improves memory performance in middle-aged mice. ${ }^{83}$

In summary, PBM increases neurotrophins, neurogenesis, synaptogenesis, and ATP, while it reduces inflammation, apoptosis, and oxidative stress. ${ }^{84}$ Through these mechanisms, PBM has the potential to be an effective treatment for MDD and comorbid disorders.

\section{Treatment Parameters and Penetration of Transcranial Photobiomodulation}

Prior to examining the effects of transcranial PBM on depressive symptoms, it is necessary to clarify that in our review the 
reported dosimetry refers to the properties of the light at the skin surface. Both the irradiance [power density: amount of electromagnetic energy (Joule) delivered per second on $1 \mathrm{~cm}^{2}$ of surface] and the fluence [energy density: amount of electromagnetic energy (Joule) delivered on $1 \mathrm{~cm}^{2}$ of surface within a given treatment session] reported for each study refer to the light at skin level. It is critical to distinguish between the light energy delivered to the skin surface and that delivered to the target tissue. In the mouse brain, the distance from skin surface to the depth of the brain is about $2 \mathrm{~mm}$. In contrast, in humans, up to 2 to $3 \mathrm{~cm}$ of intervening skin, skull, cerebrospinal fluid, and brain needs to be penetrated to adequately irradiate a target site, such as the prefrontal cortex. Therefore, it is necessary to deliver sufficient fluence to the skin surface in order for sufficient fluence to reach target areas of the human brain. A measurable power of NIR $(808 \mathrm{~nm})$ was traced at $4 \mathrm{~cm}$ from the surface of the skin in unfixed cadaver brains $(n=8)$ with a 5 -W laser device. In this experiment, the light with $808 \mathrm{~nm}$ (NIR) wavelength was superior to the 940 (NIR) and $660 \mathrm{~nm}$ (red) light in terms of penetration. ${ }^{85}$ In recently slaughtered sheep heads, a $15-\mathrm{W}$ laser device produced a $2.9 \%$ penetration of NIR $(810 \mathrm{~nm})$ at $3 \mathrm{~cm}$ from skin surface. ${ }^{86}$ Different groups have found quite different levels of penetration for LED devices. Henderson and Morries ${ }^{86}$ found that energy from a $0.2-\mathrm{W}$ LED device for red $(650 \mathrm{~nm})$ and NIR $(800 \mathrm{~nm})$ light did not penetrate $2 \mathrm{~mm}$ of ex vivo human skin. Significantly better penetration was reported by Jagdeo et al. ${ }^{87}$ who used a 0.5 -W LED device in fixed cadaver heads $(n=3)$ for NIR $(830 \mathrm{~nm})$ and claimed to deliver $2 \%$ of the light after penetrating $1 \mathrm{~cm}$ from the skin surface on frontal areas. A $2 \%$ to $3 \%$ penetration rate allows an NIR fluence on the brain equivalent to the fluence inducing neurological benefit in animal model studies. ${ }^{84}$ However, depending on parameters used, the expected brain depth of irradiation might vary from only the most superficial cortex to its whole width (including sulci), always in proximity of the light source.

\section{Transcranial Photobiomodulation in Healthy Volunteers}

In a study from the University of Texas (Austin), Barrett and Gonzalez-Lima tested the effect of transcranial PBM on memory and attention in undergraduates enrolled in an introductory psychology class. ${ }^{88}$ Forty subjects were enrolled in this double-blind, sham controlled study on NIR light. The sham acted as placebo, and consisted of just enough light to warm up the skin of the forehead with no significant irradiation to the brain. They delivered one session of NIR light to the right forehead, targeting the right frontal pole of the cerebral cortex, which is the most anterior portion of the right prefrontal cortex (Brodmann's area 9 and 10). They used the EEG sites (FP2) to center their light source toward the right frontal eminence. The instrument used was a class-IV laser CG-5000 (Cell Gen Therapeutics) and the parameters were the following: wavelength: $1064 \mathrm{~nm}$ (NIR light), irradiance: $250 \mathrm{~mW} / \mathrm{cm}^{2}$, fluence: $60 \mathrm{~J} / \mathrm{cm}^{2}$, time: 4 min per site (two sites) ${ }^{88}$ (Table 1). Similar parameters have been used clinically with comparable instruments (by Cell Gen Therapeutics) for the treatment of lower back pain, sciatica, and migraine headaches. It was estimated that $2 \%$ of the NIR light passed the cranial bone and reached the brain. The authors observed that the undergraduates who received the NIR light had faster reaction times after 2 weeks, measured by the psychomotor vigilance test. They were more attentive to the light signals on the test screen and responded more promptly. The undergraduates who underwent PBM (NIR) also performed better in terms of memory. They were able to remember more easily a distribution of red and yellow squares on the screen and after a delay they were more likely to find the right match to the initial sample (from two options); their benefits were signaled by both the greater number of correct responses and by their faster retrieval of their memory (delayed match to sample). ${ }^{88}$ Barrett and Gonzalez-Lima also observed a lasting benefit in the overall affect of the undergraduates exposed to NIR light after 2 weeks, including in several dimensions possibly related to anxiety, such as being "afraid, scared, nervous, jittery, irritable, ashamed, upset, and distressed." This was assessed by the PANAS rating scale, which explores both positive and negative affect. ${ }^{88}$ Possible confounding factors were the degree to which the undergraduates felt discouraged by the approaching finals or tests, or if they kept the enthusiasm that led them into their field of study. It is unclear from the experimental design if the effect on the overall affect was independent from the cognitive boosting effect, or if the two effects were psychologically connected.

\section{Preliminary Clinical Evidence for the Treatment of Depression with Transcranial Photobiomodulation}

To this date, there are only two preliminary open studies of transcranial PBM for the treatment of MDD that have been published. Schiffer et al. (McLean Hospital, Belmont, Massachusetts) studied 10 depressed subjects (mean HAM-D-21 total score $23.9 \pm 8.8 \mathrm{SD}$ ). The study showed good tolerability of PBM, and a single exposure of the forehead to $810 \mathrm{~nm}$ LED gave a significant reduction in depression (HAM-D scale) in just 2 weeks, despite the fact that the PBM treatment was tested on a sample of patients suffering from treatment-resistant depression. ${ }^{40}$ The treatment consisted of a single session of PBM at two sites on the forehead, aiming at the DLPFC (on EEG sites F3 and F4 bilaterally). The LED instrument used was produced by the Marubeni America Corp. and the following parameters were applied: wavelength: $810 \mathrm{~nm}$, irradiance: $250 \mathrm{~mW} / \mathrm{cm}^{2}$, fluence: $60 \mathrm{~J} / \mathrm{cm}^{2}$, and time: $4 \mathrm{~min}$ per site

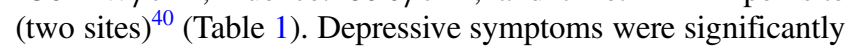
decreased at weeks 2 and 4 (delta HAM-D-21 total score 13.2 and 6.5 , respectively). The authors reported a $60 \%$ remission rate of MDD at week 2 (HAM-D-21 <10). Noticeably, depressive symptoms tended to creep back up at week 4 from the NIR treatment, suggesting that one single treatment, while sufficient in this population to produce remission, might have been inadequate to maintain it. Given the open and uncontrolled design, a placebo effect could not be assessed and ruled out; however, placebo effect tends to be marginal in patients with resistant depression.

Our group (Massachusetts General Hospital, Boston, Massachusetts) obtained preliminary data on the safety and efficacy of multiple sessions of transcranial PBM in MDD patients. ${ }^{89} \mathrm{We}$ enrolled eight adults diagnosed with MDD with at least moderate depressive symptoms. The inclusion and exclusion criteria were similar to the ones of Schiffer et al. ${ }^{40}$ We conducted a 7-week double-blind sham-controlled treatment, involving 3 weeks of NIR or sham twice a week followed by cross-over to 3 more weeks of NIR or sham, after a washout week. We reported data on the only four completers, who all received 3 weeks of active NIR followed by 3 weeks of sham. ${ }^{89}$ At each 
Cassano et al.: Review of transcranial photobiomodulation for major depressive disorder...

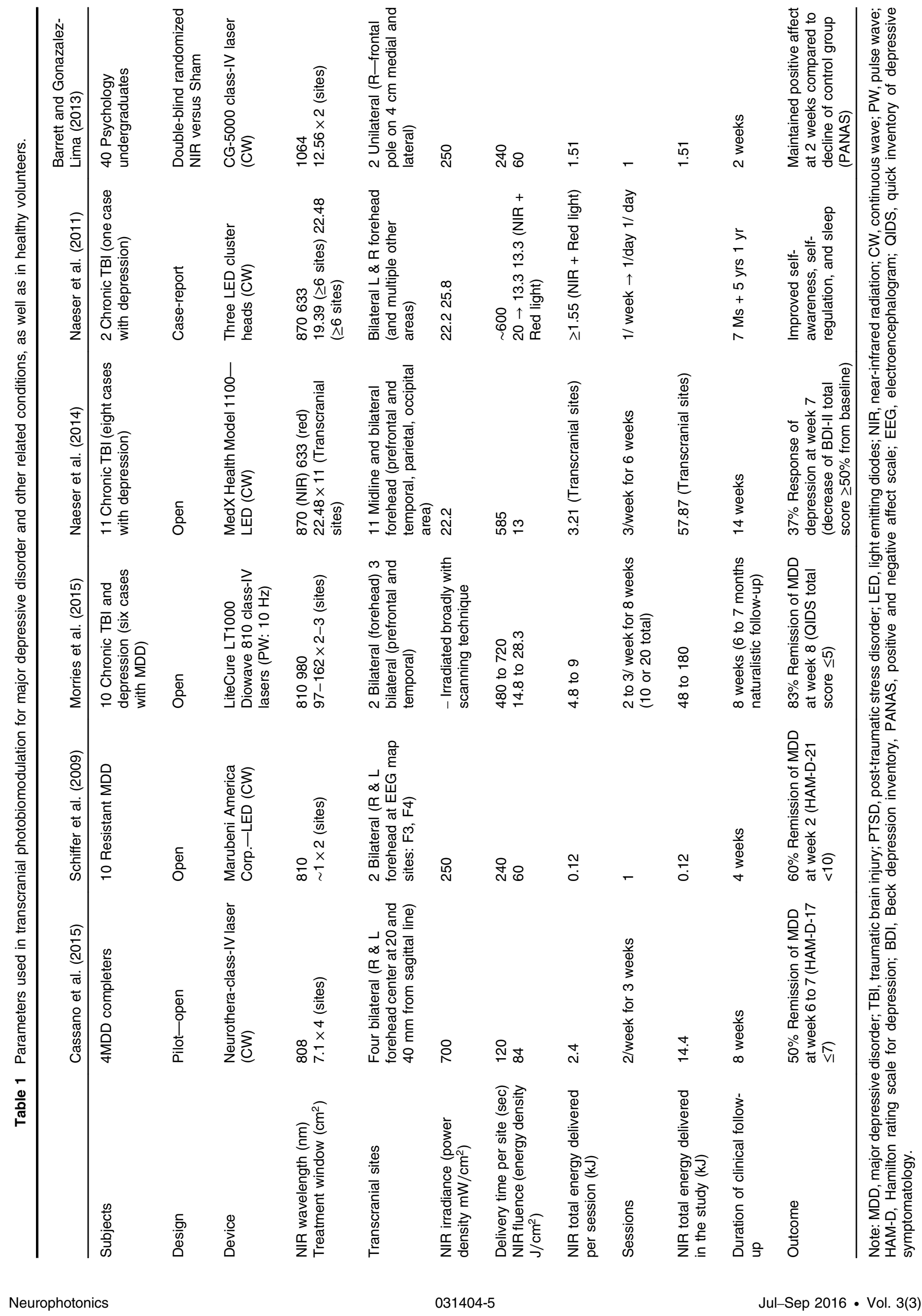


treatment session, the NIR light $(808 \mathrm{~nm})$ was administered to the forehead bilaterally at four sites $\left(2 \mathrm{~min}\right.$ per site, $12.56 \mathrm{~cm}^{2}$ spot area each). We used a laser instrument, Neurothera (classIV laser), produced by Photothera Inc. The treatment parameters were the following: NIR irradiance of $700 \mathrm{~mW} / \mathrm{cm}^{2}$ and fluence of $84 \mathrm{~J} / \mathrm{cm}^{2}$, for a total NIR energy of $2.40 \mathrm{~kJ}$ per session (Table 1). The baseline HAM-D-17 total scores averaged $19.8 \pm$ 4.35 (SD). Two of four treatment-completers (50\%) achieved remission with HAM-D-17s of 4 and 3 at weeks 6 and 7, respectively. At endpoint, the mean HAM-D-17 total scores was $13 \pm$ 5.35 (SD). The NIR treatment was well tolerated with only one adverse event (transient, mild diarrhea in a subject with irritable bowel syndrome). While our findings were limited by our small sample size, they support the hypotheses that transcranial PBM could be an effective treatment for MDD.

While most transcranial PBM treatments have been conducted with sources of continuous light, at least one group (Neuro-Laser Foundation in Denver, Colorado) has experimented with pulsed light in TBI. ${ }^{90,91}$ This contribution is potentially relevant to the field, as in animal models of TBI it appears that pulsed NIR light might be more effective than continuous wave light. ${ }^{18}$ While all 10 TBI subjects experienced symptoms of depression [Quick Inventory of Depressive Symptomatology (QIDS) mean total score $12.9 \pm 4.6 \mathrm{SD}$ ], six from this case series were also diagnosed with MDD. Morries et al. ${ }^{91}$ used class-IV lasers for transcranial PBM (LiteCure and Diowave), delivering a dual wavelength of 980 and $808 \mathrm{~nm}$ with 9 to $13 \mathrm{~W}$ average power (Table 1). Despite the high power instrument, delivering approximately double the power used by our group in Boston, Massachusetts, ${ }^{89}$ Morries et al. did not observe any thermal skin hazard, as the skin temperature increase was negligible and temperature changes (checked with a laser thermometer) never exceeded $1^{\circ} \mathrm{C}$ to $3^{\circ} \mathrm{C}$. Despite the very high irradiance, the reported fluence (14.8 to $28.3 \mathrm{~J} / \mathrm{cm}^{2}$ ) was less than what safely delivered by the Photothera device in several hundred patients. ${ }^{10-12,92}$ The high PBM irradiance used by Morries et al. was somewhat mitigated by the pulsed source, which decreases the actual time of exposure to NIR light. ${ }^{91}$ The overall time of exposure, averaging $10 \mathrm{~min}$ per site, was twice as long as the exposure time used in our clinic ( 4 min per each hemiside $^{89}$ ). However, Morries et al. also used a much larger surface on the forehead $\left(132 \mathrm{~cm}^{2}\right.$ per site on average) while aiming at the frontal cortex (roughly twice as much as the treatment area in our study). Morries et al. were able to irradiate such a wide area using a paced scanning technique, with the light source in continuous motion over the skin surface, which further explains why the actual fluence may have reportedly been maintained within a safe range. At endpoint, patients $(n=10)$ experienced a significant reduction of their depressive symptoms (mean QIDS total score $2.2 \pm 2.3 \mathrm{SD}){ }^{91}$ All six subjects with MDD responded to the treatment (decrease of QIDS total score $\geq 50 \%$ from baseline) and five remitted from depression (QIDS total score $\leq 5$ ).

Similar benefits were observed in a separate cohort of TBI patients with comorbid depression. Naeser et al. (VA Boston Health Care System) reported on a cohort of eight TBI subjects with comorbid depression who were treated with a standardized protocol of transcranial PBM. ${ }^{93}$ All patients were treated with a three LED cluster heads instrument (MedX Health Model 1100, Toronto). The parameters used for the treatment were the following: PBM wavelength: $870 \mathrm{~nm}$ (NIR) and $633 \mathrm{~nm}$ (red light), irradiance: $22.2 \mathrm{~mW} / \mathrm{cm}^{2}$, fluence: $13 \mathrm{~J} / \mathrm{cm}^{2}$, time: $\sim 10 \mathrm{~min}$ $(585 \mathrm{sec})$ per site. The NIR light was applied three times per week for 6 weeks (18 sessions), on 11 sites for about $10 \mathrm{~min}$ per site (the total duration of each session was $20 \mathrm{~min}$ ). ${ }^{93}$ The sites on the skull were chosen on the midline from front to back, and bilaterally on frontal, parietal, and temporal areas; the cortical targets included the DLPFC and the central executive network (Table 1). The eight subjects were identified as having mild, moderate, or severe depression based on the BDI-II total score (mean BDI-II total scores were $22 \pm 7.4$ SD). Three subjects $(37 \%)$ showed antidepressant response (decrease of BDI-II total score $\geq 50 \%$ from baseline) after 6 weeks of PBM treatment (mean BDI-II total scores were $14.8 \pm 6.5 \mathrm{SD}$ ). Two of them maintained the antidepressant response after 8 additional weeks of follow-up. ${ }^{93}$ The lower response rates observed, compared to the prior studies in depressed subjects, could be related to the comorbidity with TBI, to the study sample, or to the different technique and parameters used for transcranial PBM.

\section{Preliminary Clinical Evidence for the Treatment of Psychiatric Comorbidity with Transcranial Photobiomodulation}

\subsection{Traumatic Brain Injury}

While only one randomized controlled trial (RCT) has been published assessing the impact on cognition of transcranial PBM (see Section 8), several reports have shown its therapeutic effects in TBI patients with highly comorbid depression.

Two case reports in patients with TBI suggested that transcranial PBM improved sustained attention, memory, and executive functions. Both patients were treated with an instrument with three separate LED cluster heads. The parameters used for the treatment were the following: wavelength: 870 (NIR) and $633 \mathrm{~nm}$ (red light), irradiance: 22.2 to $25.8 \mathrm{~mW} / \mathrm{cm}^{2}$, fluence: $13.3 \mathrm{~J} / \mathrm{cm}^{2}$, time: $10 \mathrm{~min}$ per site (Table 1). ${ }^{94}$ While the irradiance was one order of magnitude less than the one used in healthy volunteers ${ }^{88}$ the exposure time was about one order of magnitude longer and three to five times as many sites were irradiated ${ }^{94}$ Factoring in also the larger surface of irradiation (greater number of sites), it is likely that the actual energy delivered to the scalp was similar for the TBI patients to what was delivered to the healthy volunteers by Barrett and GonzalezLima. ${ }^{88}$ Notably, symptoms began to recur if the patients did not maintain daily PBM treatments. This may reflect the relatively minute amounts of photonic energy that penetrated skin and scalp to reach the surface of the brain.

The same group, Naeser et al. ${ }^{93}$ reported on a cohort of 11 subjects with chronic mild TBI who were treated with a very similar PBM protocol (Table 1). The 11 subjects presented persistent cognitive dysfunction. Neuropsychological testing was performed at baseline, after 6 weeks of NIR treatment and after 4 and 8 weeks of additional follow-up. A significant effect was observed for transcranial NIR treatment over time on attention, inhibition and inhibition switching at the Stroop task (Trial 3 and 4). ${ }^{93}$ Naeser et al. also observed a significant improvement of verbal learning and memory, as well as on long-delay free recall at the California Verbal Learning Test (CVLT)-II. Participants and family reported better ability to perform social, interpersonal, and occupational functions in treated subjects. ${ }^{93}$

Similar functional improvements were reported by Morries et al. in 10 patients with chronic TBI treated with transcranial pulse PBM (Table 1), including improvement of work 
performance or return to work and overall dramatic improvements of quality of life, assessed by the patient (diary), by family members (spousal diary), and by the treating clinician. ${ }^{91}$

\subsection{Anxiety and Post-Traumatic Stress Syndrome}

In the aforementioned cohorts, anxiety disorders were also frequently comorbid. Similar to depressive manifestations, anxiety symptoms appeared to respond to PBM. Schiffer et al. ${ }^{40}$ treated nine subjects with comorbid anxiety and traumatic stress disorders (mean HAM-A total score $23.0 \pm 12.2 \mathrm{SD}$ ) with transcranial PBM (Table 1). The study showed a significant improvement in anxiety at weeks 2 and 4 (delta HAM-A total score were 14.9 and 9 , respectively). ${ }^{40}$ Further, all three patients with PTSD were among those achieving significant anxiety reduction by week 2 (HAM-A total score decrease $\geq 50 \%$ ).

One of the two cases with chronic TBI reported by Naeser et al. was a 52-year-old veteran with comorbid PTSD. After few months of treatment with transcranial PBM (Table 1), she noticed improvement in self-awareness and self-regulation in both social and work milieus. These benefits were attributed to improved sleep and reduced impulsivity, irritation and anger, consistent with common PTSD symptoms. ${ }^{94}$ After 4 months of treatment her medical disability was discontinued and she returned to her prior work..$^{94}$ In Naeser et al.$^{93}$ there was a cohort of 11 patients with chronic TBI, and all four patients with PTSD had clinically meaningful reductions in related symptoms (Table 1). These preliminary clinical data are in line with a report from Rojas et al. ${ }^{95}$ on the therapeutic effects of PBM on anxiety and post-traumatic syndromes. Transcranial PBM (660 nm) improved fear extinction and lessened fear recall in a rat model of fear conditioning and extinction. ${ }^{95}$

\subsection{Insomnia}

Transcranial PBM (658 nm) has been demonstrated to improve sleep quality in Chinese female basketball players. Zhao et al., ${ }^{96}$ at the China Institute of Sports Science in Beijing, randomly assigned 20 athletes to receive red light or sham. The light was administered for $30 \mathrm{~min}$ for 14 consecutive nights. A whole-body red-light treatment machine (Shanghai Dayou PDT Technology Co, Ltd., Shanghai, China) with a fluence of $30 \mathrm{~J} / \mathrm{cm}^{2}$ was used. Sleep quality improved significantly with this intervention, as measured by the Pittsburgh Sleep Quality Index, as well as serum melatonin levels, and endurance performance of the players. ${ }^{96}$ While the aforementioned study used a whole body irradiation instead of just transcranial irradiation, some inferences can be made on the possible role of transcranial PBM in the treatment of sleep disturbances.

The case-report and case-series (already mentioned above) discussing the treatment of chronic TBI with transcranial PBM also suggests a possible hypnotic effect (Table 1). ${ }^{91,93,94} \mathrm{~A}$ confounding factor for our interpretation of these effects of transcranial PBM on sleep is the high prevalence of comorbid mood and anxiety disorders, as well as the primary diagnosis of TBI. To our knowledge, there are no published data on the hypnotic effect of transcranial PBM in primary insomnia.

\subsection{Suicidal Ideation}

Preliminary data from our group suggest a possible therapeutic effect of transcranial PBM on suicidal ideation. ${ }^{89}$ In our cohort of four treatment-completers, two subjects achieved remission from MDD and from suicidal ideation (i.e., the Concise Health Risk Tracking scale-CHRT-scores decreased from 28 to 5 and from 17 to 2, respectively, at weeks 6 and 7). Morries et al. also found that TBI subjects $(n=10)$ treated with transcranial PBM (Table 1) had a remarkable improvement in suicidal ideation. ${ }^{91}$ All patients denied any suicidal thoughts at endpoint, including two and three who were previously thinking of suicide or death "several times a day in depth" and "several times a week for several minutes," respectively (QIDS item $\# 12$ = 3 and 2). The mean QIDS item \#12 was $1.4 \pm 1.2 \mathrm{SD}$ at baseline and 0 at endpoint $(n=10 ; p=0.002$; unpublished data). If confirmed in a larger sample of at risk subjects, the therapeutic effect of transcranial PBM on suicidal ideation could stem from its antidepressant and anxiolytic effects, as well as from its effects on cognition. ${ }^{97}$

\section{Appraising the Literature on Transcranial Photobiomodulation}

Overall, our paper is extracting information from very few studies. The number of actual studies of PBM on MDD is very small, inclusive of very few actual participants. Therefore, the evidence in the literature for the treatment of MDD and related conditions with transcranial PBM is very preliminary. Also, it is at times difficult to extricate the effects on mood, anxiety, cognition, sleep, and suicidal ideation, as these constructs are inexorably linked in the populations under study. In the spirit of completeness, we have included sections covering suicidal ideation and insomnia, despite sparse evidence in regards of the use of transcranial PBM for these conditions. Highlighting all these conditions might help defining future areas of investigation in the field of psychiatric PBM. Future studies should include sham treatments when exploring the therapeutic effects of transcranial PBM, given high placebo effect associated with device procedures $^{98}$ and with frequent clinical visits. ${ }^{99}$ An adequate sham-controlled study should be using only invisible light (NIR as opposed to red light) and either adequate cooling in both treatment arms or a source of heat for the sham to prevent unblinding.

\section{Conclusions}

Transcranial PBM is safe and well-tolerated. While there are no documented incidents of retinal or skin injury in patients treated with transcranial PBM, patients generally wear eye protections whenever receiving laser light. The preliminary data reviewed in this paper suggest the efficacy of transcranial PBM for the treatment of depression, anxiety, and cognitive impairment. In the absence of RCTs - testing the safety and efficacy of transcranial PBM for psychiatric disorders-scientific skepticism remains appropriate. This review paper is intended to draw more attention from the scientific community toward a promising field in need of further research. Given the low cost of LED devices and the FDA approval for their over-the-counter use, there is potential for wide dissemination of transcranial PBM, if proven to be safe and effective in psychiatric patients. Given the potential risk of injury when high-powered NIR devices are used by untrained personnel or laypersons, over-the-counter availability is not recommended for devices emitting greater than $1 \mathrm{~W}$.

\section{Acknowledgments}

The authors would like to thank Dr. Larry Morries for sharing his raw data on depressive outcomes in TBI subjects for additional analyses on suicidality, presented in this paper. 
Financial Disclosures: Dr. P. Cassano's salary was supported by the Harvard Psychiatry Department (DupontWarren Fellowship and Livingston Award), by the Brain and Behavior Research Foundation (NARSAD Young Investigator Award), and by the Photothera Inc. unrestricted grant. Drug donation from TEVA. Travel reimbursement from PharmaciaUpjohn. Dr. M. Hamblin was supported by US NIH Grant No. R01AI050875, by Air Force Office of Scientific Research Grant No. FA9550-13-1-0068, by US Army Medical Research Acquisition Activity Grant No. W81XWH-09-1-0514, and by US Army Medical Research and Materiel Command Grant No. W81XWH-13-2-0067. Research grants and consulting fees from Photothera Inc. Scientific Advisory Board of Transdermal Cap Inc (LLLT for hair regrowth). Dr. D. Iosifescu: In the last three years, he has been a consultant for Avanir, CNS Response, INSYS Therapeutics, Lundbeck, Otsuka, Servier, Sunovion and has received research support through Mount Sinai from Alkermes, Astra Zeneca, Brainsway, Euthymics, Neosync, Roche, and Shire. Theodore A. Henderson MD, PhD, is president of The Synaptic Space, a medical consulting firm. He is president of Dr. Theodore Henderson, Inc., a clinical service firm. He is co-owner of Neuro-Luminance, a clinical service organization. He is president of the International Society of Applied Neuroimaging. He is vice-president of the Neuro-Laser Foundation, a nonprofit foundation. Mr. S. Petrie reported no financial disclosures.

\section{References}

1. I. Kochevar, M. Pathak, and J. Parrish, "Photophysics, photochemistry, and photobiology," in Fitzpatrick's Dermatology in General Medicine, pp. 220-229, McGraw-Hill, New York (1999).

2. P. Schroeder et al., "Photoprotection beyond ultraviolet radiationeffective sun protection has to include protection against infrared A radiation-induced skin damage," Skin Pharmacol. Physiol. 23(1), 15-17 (2010).

3. C. Ferraresi, M. R. Hamblin, and N. A. Parizotto, "Low-level laser (light) therapy (LLLT) on muscle tissue: performance, fatigue and repair benefited by the power of light," Photonics Lasers Med. 1(4), 267-286 (2012).

4. M. E. Chaves et al., "Effects of low-power light therapy on wound healing: LASER x LED," An. Bras. Dermatol. 89(4), 616-623 (2014).

5. L. B. Harkless et al., "Improved foot sensitivity and pain reduction in patients with peripheral neuropathy after treatment with monochromatic infrared photo energy-MIRE," J. Diabetes Complications 20(2), 81-87 (2006).

6. G. Allais et al., "Non-pharmacological approaches to chronic headaches: transcutaneous electrical nerve stimulation, lasertherapy and acupuncture in transformed migraine treatment," Neurol. Sci. 24(Suppl. 2), S138-S142 (2003).

7. B. Hersant et al., "Current indications of low-level laser therapy in plastic surgery: a review," Photomed. Laser Surg. 33(5), 283-297 (2015).

8. B. A. Russell, N. Kellett, and L. R. Reilly, "A study to determine the efficacy of combination LED light therapy $(633 \mathrm{~nm}$ and $830 \mathrm{~nm})$ in facial skin rejuvenation," J. Cosmet. Laser Ther. 7(3-4), 196-200 (2005).

9. D. T. Delpy et al., "Cerebral monitoring in newborn infants by magnetic resonance and near infrared spectroscopy," Scand. J. Clin. Lab. Invest. Suppl. 47(188), 9-17 (1987).

10. P. A. Lapchak et al., "Transcranial near-infrared light therapy improves motor function following embolic strokes in rabbits: an extended therapeutic window study using continuous and pulse frequency delivery modes," Neuroscience 148(4), 907-914 (2007).

11. P. A. Lapchak, J. Wei, and J. A. Zivin, "Transcranial infrared laser therapy improves clinical rating scores after embolic strokes in rabbits," Stroke 35(8), 1985-1988 (2004).

12. A. Oron et al., "Low-level laser therapy applied transcranially to rats after induction of stroke significantly reduces long-term neurological deficits," Stroke 37(10), 2620-2624 (2006).
13. L. Detaboada et al., "Transcranial application of low-energy laser irradiation improves neurological deficits in rats following acute stroke," Lasers Surg. Med. 38(1), 70-73 (2006).

14. Y. Lampl, "Laser treatment for stroke," Expert Rev Neurother. 7(8), 961-965 (2007).

15. W. Hacke et al., "Transcranial laser therapy in acute stroke treatment results of neurothera effectiveness and safety trial 3, a phase III clinical end point device trial," Stroke 45(11), 3187-3193 (2014).

16. Y. Lampl et al., "Infrared laser therapy for ischemic stroke: a new treatment strategy results of the neurothera effectiveness and safety trial-1 (NEST-1)," Stroke 38(6), 1843-1849 (2007).

17. J. A. Zivin et al., "Effectiveness and safety of transcranial laser therapy for acute ischemic stroke," Stroke 40(4), 1359-1364 (2009).

18. T. Ando et al., "Comparison of therapeutic effects between pulsed and continuous wave 810-nm wavelength laser irradiation for traumatic brain injury in mice," PLoS One 6(10), e26212-e26220 (2011).

19. Y. Y. Huang et al., "Transcranial low level laser (light) therapy for traumatic brain injury," J. Biophotonics 5, 827-837 (2012).

20. W. Xuan et al., "Low-level laser therapy for traumatic brain injury in mice increases brain derived neurotrophic factor (BDNF) and synaptogenesis," J. Biophotonics 9999(9999) 502-511 (2014).

21. W. Xuan et al., "Transcranial low-level laser therapy enhances learning, memory, and neuroprogenitor cells after traumatic brain injury in mice," J. Biomed. Opt. 19(10), 108003 (2014).

22. W. Xuan et al., "Transcranial low-level laser therapy improves neurological performance in traumatic brain injury in mice: effect of treatment repetition regimen," PLoS One 8(1), e53454 (2013).

23. M. A. Naeser and M. R. Hamblin, "Potential for transcranial laser or LED therapy to treat stroke, traumatic brain injury, and neurodegenerative disease," Photomed. Laser Surg. 29(7), 443-446 (2011).

24. M. A. Naeser et al., "Improved cognitive function after transcranial, light-emitting diode treatments in chronic, traumatic brain injury: two case reports," Photomed. Laser Surg. 29(5), 351-358 (2011).

25. M. A. Naeser et al., "Significant improvements on cognitive performance post-transcranial, red/near-infrared LED treatments in chronic, mild TBI: open-protocol study," J. Neurotrauma 31(11), 1008-1017 (2014).

26. S. Purushothuman et al., "The impact of near-infrared light on dopaminergic cell survival in a transgenic mouse model of parkinsonism," Brain Res. 1535, 61-70 (2013).

27. L. De Taboada et al., "Transcranial laser therapy attenuates amyloidbeta peptide neuropathology in amyloid-beta protein precursor transgenic mice," J. Alzheimers Dis. 23(3), 521-535 (2011).

28. E. A. Kim et al., "Macular degeneration in an arc welder," Ind. Health," 45(2), 371-373 (2007).

29. C. M. Moore et al., "Lower levels of nucleoside triphosphate in the basal ganglia of depressed subjects: a phosphorous-31 magnetic resonance spectroscopy study," Am. J. Psychiatry 154(1), 116-118 (1997).

30. H.-P. Volz et al., "31P magnetic resonance spectroscopy in the frontal lobe of major depressed patients," Eur. Arch. Psychiatry Clin. Neurosci. 248(6), 289-295 (1998).

31. P. F. Renshaw et al., "Multinuclear magnetic resonance spectroscopy studies of brain purines in major depression," Am. J. Psychiatry 158(12), 2048-2055 (2001).

32. D. V. Iosifescu et al., "Brain bioenergetics and response to triiodothyronine augmentation in major depressive disorder," Biol. Psychiatry 63(12), 1127-1134 (2008).

33. P. Videbech, "PET measurements of brain glucose metabolism and blood flow in major depressive disorder: a critical review," Acta Psychiatr. Scand. 101(1), 11-20 (2000).

34. H. S. Mayberg et al., "Regional metabolic effects of fluoxetine in major depression: serial changes and relationship to clinical response," Biol. Psychiatry 48(8), 830-843 (2000).

35. W. C. Drevets, W. Bogers, and M. E. Raichle, "Functional anatomical correlates of antidepressant drug treatment assessed using PET measures of regional glucose metabolism," Eur. Neuropsychopharmacol. 12(6), 527-544 (2002).

36. S. Kennedy et al., "Differences in brain glucose metabolism between responders to CBT and venlafaxine in a 16-week randomized controlled trial," Am. J. Psychiatry 164(5), 778-788 (2007).

37. W. Yu et al., "Photomodulation of oxidative metabolism and electron chain enzymes in rat liver mitochondria," Photochem. Photobiol. 66(6), 866-871 (1997). 
38. N. Mochizuki-Oda et al., "Effects of near-infra-red laser irradiation on adenosine triphosphate and adenosine diphosphate contents of rat brain tissue," Neurosci. Lett. 323(3), 207-210 (2002).

39. U. Oron et al., "Ga-As (808 nm) laser irradiation enhances ATP production in human neuronal cells in culture," Photomed. Laser Surg. 25(3), 180-182 (2007).

40. F. Schiffer et al., "Psychological benefits 2 and 4 weeks after a single treatment with near infrared light to the forehead: a pilot study of 10 patients with major depression and anxiety," Behav. Brain Funct. 5, 46 (2009).

41. R. Shelton et al., "Altered expression of genes involved in inflammation and apoptosis in frontal cortex in major depression," Mol. Psychiatry 16(7), 751-762 (2010).

42. N. Simon et al., "A detailed examination of cytokine abnormalities in major depressive disorder," Eur. Neuropsychopharmacol. 18(3), 230233 (2008).

43. C. L. Raison, L. Capuron, and A. H. Miller, "Cytokines sing the blues: inflammation and the pathogenesis of depression," Trends Immunol. 27(1), 24-31 (2006).

44. I. Goshen et al., "Brain interleukin-1 mediates chronic stress-induced depression in mice via adrenocortical activation and hippocampal neurogenesis suppression," Mol. Psychiatry 13(7), 717-728 (2007).

45. D. Sasayama et al., "Increased cerebrospinal fluid interleukin-6 levels in patients with schizophrenia and those with major depressive disorder," J. Psychiatr. Res. 47(3), 401-406 (2013).

46. D. Lindqvist et al., "Interleukin-6 is elevated in the cerebrospinal fluid of suicide attempters and related to symptom severity," Biol. Psychiatry 66(3), 287-292 (2009).

47. J. Levine et al., "Cerebrospinal cytokine levels in patients with acute depression," Neuropsychobiology 40(4), 171-176 (1999).

48. H. Araki et al., "Reduction of interleukin-6 expression in human synoviocytes and rheumatoid arthritis rat joints by linear polarized near infrared light (Superlizer) irradiation," Laser Ther. 20(4), 293 (2011).

49. M. Yamaura et al., "Low level light effects on inflammatory cytokine production by rheumatoid arthritis synoviocytes," Lasers Surg. Med. 41(4), 282-290 (2009).

50. J. Khuman et al., "Low-level laser light therapy improves cognitive deficits and inhibits microglial activation after controlled cortical impact in mice," J. Neurotrauma 29(2), 408-417 (2012).

51. D. Barolet and A. Boucher, "Radiant near infrared light emitting diode exposure as skin preparation to enhance photodynamic therapy inflammatory type acne treatment outcome," Lasers Surg. Med. 42(2), 171178 (2010).

52. A. Sarandol et al., "Major depressive disorder is accompanied with oxidative stress: short-term antidepressant treatment does not alter oxidative-antioxidative systems," Hum. Psychopharmacol. 22(2), 6773 (2007)

53. İ. Eren, M. Nazıroğlu, and A. Demirdaş, "Protective effects of lamotrigine, aripiprazole and escitalopram on depression-induced oxidative stress in rat brain," Neurochem. Res. 32(7), 1188-1195 (2007).

54. J. D. Hayes and L. I. McLellan, "Glutathione and glutathione-dependent enzymes represent a co-ordinately regulated defence against oxidative stress," Free Radical Res. 31(4), 273-300 (1999).

55. D. C. Shungu et al., "Increased ventricular lactate in chronic fatigue syndrome. III. Relationships to cortical glutathione and clinical symptoms implicate oxidative stress in disorder pathophysiology," NMR Biomed. 25(9), 1073-1087 (2012).

56. D. Del Rio, A. J. Stewart, and N. Pellegrini, "A review of recent studies on malondialdehyde as toxic molecule and biological marker of oxidative stress," Nutr. Metab. Cardiovasc. Dis. 15(4), 316-328 (2005).

57. J. A. Tainer et al., "Structure and mechanism of copper, zinc superoxide dismutase," Nature 306, 284-287 (1983).

58. M. E. Ozcan et al., "Antioxidant enzyme activities and oxidative stress in affective disorders," Int. Clin. Psychopharmacol. 19(2), 89-95 (2004).

59. P. Chelikani, I. Fita, and P. Loewen, "Diversity of structures and properties among catalases," Cell. Mol. Life Sci. 61(2), 192-208 (2004).

60. S. Shi et al., "Protective effect of nitric oxide against oxidative stress under ultraviolet-B radiation," Nitric Oxide 13(1), 1-9 (2005).

61. D. A. Wink et al., "Nitric oxide protects against alkyl peroxide-mediated cytotoxicity: further insights into the role nitric oxide plays in oxidative stress," Arch. Biochem. Biophys. 319(2), 402-407 (1995).
62. İ. Eren et al., "Venlafaxine modulates depression-induced oxidative stress in brain and medulla of rat," Neurochem. Res. 32(3), 497-505 (2007).

63. C. F. Rizzi et al., "Effects of low-level laser therapy (LLLT) on the

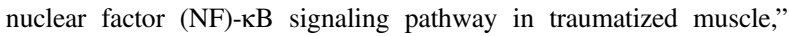
Lasers Surg. Med. 38(7), 704-713 (2006).

64. I. N. Mungrue, M Husain, and D. J. Stewart, "The role of NOS in heart failure: lessons from murine genetic models," Heart Failure Rev. 7(4), 407-422 (2002).

65. L. Chludzińska et al., "Near-infrared radiation protects the red cell membrane against oxidation," Blood Cells Mol. Dis. 35(1), 74-79 (2005).

66. A. Dranovsky and R. Hen, "Hippocampal neurogenesis: regulation by stress and antidepressants," Biol. Psychiatry 59(12), 1136-1143 (2006).

67. R. S. Duman and L. M. Monteggia, "A neurotrophic model for stressrelated mood disorders," Biol. Psychiatry 59(12), 1116-1127 (2006).

68. G. Kempermann and G. Kronenberg, "Depressed new neurons?-adult hippocampal neurogenesis and a cellular plasticity hypothesis of major depression," Biol. Psychiatry 54(5), 499-503 (2003).

69. B. Jacobs, H. Van Praag, and F. Gage, "Adult brain neurogenesis and psychiatry: a novel theory of depression," Mol. Psychiatry 5(3), 262269 (2000).

70. S. Campbell and G. MacQueen, "The role of the hippocampus in the pathophysiology of major depression," J. Psychiatry Neurosci. 29(6), 417-426 (2004).

71. M. R. Drew and R. Hen, "Adult hippocampal neurogenesis as target for the treatment of depression," CNS Neurol. Disord. Drug Targets 6(3), 205-218 (2007).

72. A. Sahay and R. Hen, "Adult hippocampal neurogenesis in depression," Nat. Neurosci. 10(9), 1110-1115 (2007).

73. B. Vollmayr, M. M. Mahlstedt, and F. A. Henn, "Neurogenesis and depression: what animal models tell us about the link," Eur. Arch. Psychiatry Clin. Neurosci. 257(5), 300-303 (2007).

74. D. J. David et al., "Neurogenesis-dependent and-independent effects of fluoxetine in an animal model of anxiety/depression," Neuron 62(4), 479-493 (2009).

75. L. Santarelli et al., "Requirement of hippocampal neurogenesis for the behavioral effects of antidepressants," Science 301(5634), 805-809 (2003).

76. A. Giuliani et al., "Low infra red laser light irradiation on cultured neural cells: effects on mitochondria and cell viability after oxidative stress," BMC Complement. Altern. Med. 9(1), 8 (2009).

77. J. C. Rojas et al., "Neuroprotective effects of near-infrared light in an in vivo model of mitochondrial optic neuropathy," J. Neurosci. 28(50), 13511-13521 (2008).

78. J. Eells et al., "Therapeutic photobiomodulation for methanol-induced retinal toxicity," Proc. Natl. Acad. Sci. U. S. A. 100(6), 3439-3444 (2003).

79. M. T. Wong-Riley et al., "Photobiomodulation directly benefits primary neurons functionally inactivated by toxins role of cytochrome c oxidase," J. Biol. Chem. 280(6), 4761-4771 (2005).

80. W. Xuan et al., "Transcranial low-level laser therapy improves neurological performance in traumatic brain injury in mice: effect of treatment repetition regimen," PLoS One 8(1), e53454 (2013).

81. Q. Wu et al., "Low-level laser therapy for closed-head traumatic brain injury in mice: effect of different wavelengths," Lasers Surg. Med. 44(3), 218-226 (2012).

82. A. Oron et al., "Low-level laser therapy applied transcranially to mice following traumatic brain injury significantly reduces long-term neurological deficits," J. Neurotrauma 24(4), 651-656 (2007).

83. S. Michalikova et al., "Emotional responses and memory performance of middle-aged CD1 mice in a 3D maze: effects of low infrared light," Neurobiol. Learning Mem. 89(4), 480-488 (2008).

84. H. Chung et al., "The nuts and bolts of low-level laser (light) therapy," Ann. Biomed. Eng. 40(2), 516-533 (2012).

85. C. E. Tedford et al., "Quantitative analysis of transcranial and intraparenchymal light penetration in human cadaver brain tissue," Lasers Surg. Med. 47(4), 312-322 (2015).

86. T. A. Henderson and L. D. Morries, "Near-infrared photonic energy penetration- can infrared phototherapy effectively reach the human brain?," Neuropsychiatr. Dis. Treat. 11, 2191-2208 (2015). 
87. J. R. Jagdeo et al., "Transcranial red and near infrared light transmission in a cadaveric model," PLoS One 7(10), e47460 (2012).

88. D. Barrett and F. Gonzalez-Lima, "Transcranial infrared laser stimulation produces beneficial cognitive and emotional effects in humans," Neuroscience 230, 13-23 (2013).

89. P. Cassano et al., "Near-infrared transcranial radiation for major depressive disorder: proof of concept study," Psychiatry J. 2015, 352979 (2015).

90. T. A. Henderson and L. D. Morries, "SPECT perfusion imaging demonstrates improvement of TBI with transcranial near infrared laser phototherapy," Adv. Mind Body Med. 29, 27-33 (2015).

91. L. D. Morries, P. Cassano, and T. A. Henderson, "Treatments for traumatic brain injury with emphasis on transcranial near infrared laser phototherapy," Neuropsychiatr. Dis. Treat. 11, 2159-2175 (2015).

92. J. Zivin et al., "NeuroThera ${ }^{\circledR}$ efficacy and safety trial-3 (NEST-3): a double-blind, randomized, sham-controlled, parallel group, multicenter, pivotal study to assess the safety and efficacy of transcranial laser therapy with the NeuroThera ${ }^{\circledR}$ Laser System for the treatment of acute ischemic stroke within $24 \mathrm{~h}$ of stroke onset," Int. J. Stroke 9(7), 950-955 (2012).

93. M. A. Naeser et al., "Significant improvements in cognitive performance post-transcranial, red/near-infrared light-emitting diode treatments in chronic, mild traumatic brain injury: open-protocol study," J. Neurotrauma 31(11), 1008-1017 (2014).

94. M. A. Naeser et al., "Improved cognitive function after transcranial, light-emitting diode treatments in chronic, traumatic brain injury: two case reports," Photomed. Laser Surg. 29(5), 351-358 (2011).

95. J. C. Rojas, A. K. Bruchey, and F. Gonzalez-Lima, "Low-level light therapy improves cortical metabolic capacity and memory retention," J. Alzheimers Dis. 32(3), 741-752 (2012)

96. J. Zhao et al., "Red light and the sleep quality and endurance performance of Chinese female basketball players," J. Athl. Train. 47(6), 673 (2012).

97. J. G. Keilp et al., "Neuropsychological dysfunction in depressed suicide attempters," Am. J. Psychiatry 158(5), 735-741 (2001).

98. T. J. Kaptchuk et al., "Do medical devices have enhanced placebo effects?," J. Clin. Epidemiol. 53(8), 786-792 (2000).

99. M. A. Posternak and M. Zimmerman, "Therapeutic effect of follow-up assessments on antidepressant and placebo response rates in antidepressant efficacy trials Meta-analysis," Br. J. Psychiatry 190(4), 287-292 (2007).
Paolo Cassano, MD, PhD, is a principal investigator at the Center for Anxiety and Traumatic Stress Disorders and at the Depression Clinical and Research Program, Massachusetts General Hospital; and an assistant professor at Harvard Medical School. He specializes in photomodulation for psychiatric disorders. In 2015, his work on photomodulation was featured by the Boston Globe as one of the breakthroughs for the toughest medical conditions. He was also interviewed by CNN and by the Washington Post.

Samuel R. Petrie is the administrative research coordinator at the Depression Clinical and Research Program at Massachusetts General Hospital. His primary research interest is alternative therapies for mood and anxiety disorders.

Michael R. Hamblin, $\mathrm{PhD}$, is a principal investigator at the Wellman Center for Photomedicine, Massachusetts General Hospital, an associate professor at Harvard Medical School and affiliated faculty at HST-MIT. His research interests are in photodynamic therapy and photobiomodulation. He has published over 300 peer-reviewed articles and 20 textbooks. He is the associate editor for nine journals, serves on NIH Study-Sections, and in 2011 was honored by election as a fellow of SPIE.

Theodore A. Henderson is a president of both The Synaptic Space and Dr. Theodore Henderson, Inc., clinical service firms. He is cofounder of both Neuro-Luminance, a clinical service organization, and the Neuro-Laser Foundation, a nonprofit foundation. $\mathrm{He}$ is a president of the International Society of Applied Neuroimaging. He has published in neuroimaging, psychopharmacology, dementia, photobiomodulation, and traumatic brain injury. He is the editor or guest editor for numerous journals. The work of his and his colleagues was recently recognized as a "Top Science Story" by Discover Magazine for 2015.

Dan V. losifescu, MD, MSc, is a director of the Adult Psychopharmacology Program and associate professor of psychiatry and neuroscience at the Icahn School of Medicine at Mount Sinai. His research is focused on treatments for mood disorders (such as ketamine and magnetic stimulation), and on neuroimaging (MRI, MRS), and neurophysiology (quantitative EEG) studies of brain abnormalities in mood disorders. He has authored more than 125 peer-reviewed articles and 14 book chapters. 